\title{
Insights into the Eco-Friendly Adsorption of Caffeine from Contaminated Solutions by Using Hydrogel Beads
}

Aharon Zarzar ${ }^{1}$, Minyeong Hong ${ }^{1}$, Bertha $\mathbf{P}$ Llanos $^{2}$ and Abel E Navarro ${ }^{1 *}$

${ }^{1}$ Science Department, Borough of Manhattan Community College, City University of New York, New York, USA.

${ }^{2}$ Seccion Química, Departamento de Ciencias Exactas, Facultad de Ciencias y Filosofía, Universidad Peruana Cayetano Heredia, Perú.

\begin{abstract}
The presence of drugstore products in water streams has become a major concern in environmental remediation. Preliminary studies have reported that caffeine has negative impacts in animals and ecology in general. Bioremediation emphasizes on the elimination of heavy metals and toxic organic pollutants, but little attention has been paid to the so-called pollutants of emerging concern (i.e. caffeine). This research evaluates the role of $\mathrm{pH}$, adsorbent dose, salinity and time on the elimination of caffeine from aqueous solutions by using chitosan and alginate hydrogel beads. Experimental data indicates a strong $\mathrm{pH}$ effect on the adsorption, showing its highest values around neutral conditions. On the other hand, salinity has a negative effect on the adsorption, due to competition for the adsorption sites of both hydrogels. Finally, time dependence experiments demonstrate that less than 20 minutes are needed to reach adsorption equilibrium. These results suggest that biodegradable and cost-effective materials are good candidates for the removal of pollutants of emerging concern from contaminated waters.
\end{abstract}

Keywords: Hydrogel beads; Alginate; Chitosan; Caffeine; Adsorption

\section{Introduction}

Application of Green Chemistry in environmental decontamination has recently become an important alternative in the world. However, the pollution that is produced by previous generations is a latent problem that our generation faces today. Caffeine is an alkaloid (Figure 1) that is commonly used to improve mental alertness. Caffeine works as a central nervous system stimulator (CNS), which can increase blood flow, amongst other symptoms [1]. A major problem is the increase of caffeine concentrations in bodies of water such as the Pacific Ocean. The effects on sea life are still unknown, but considering the effects it has on humans, it would not be surprising that caffeine also has effects of sea life [2]. A sustainable solution would be to adsorb caffeine by a selective, yet inexpensive adsorbent from the wastewaters of the major industrial producers. Currently, there is no action being taken to resolve this growing problem, resulting in the lack of regulation for caffeine discharges in water. However, this latent problem is present in our environment, waiting for the catastrophic effects to be unfolded.

Caffeine (1,3,7-trimethylxanthineis) is a chemical compound that alters the CNS and acts as a diuretic, found in coffee beans, tea leaves, cacao pods and more than sixty other plants. Caffeine is a major drug used in the world, consumed by approximately ninety percent of the world population [3]. Caffeine gets absorbed and sent to the brain, to expose its physiological activity. However it is rapidly eliminated in the urine [4]. It is important to highlight that human body has no nutritional need for caffeine, and could survive without it $[1,4]$.

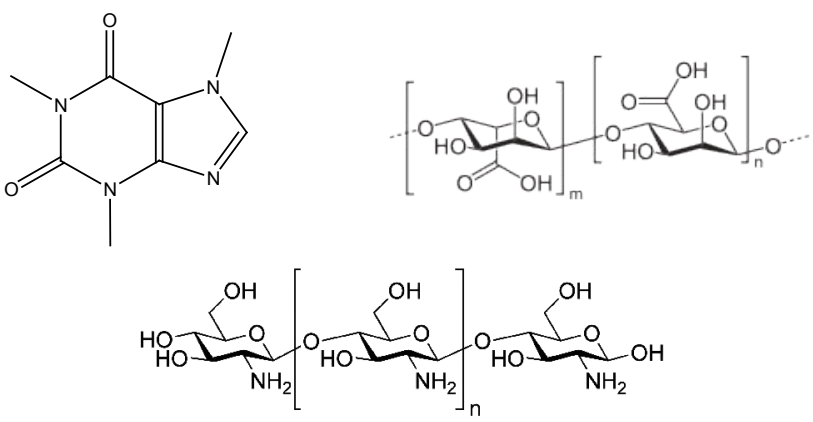

Figure 1: Chemical structures of caffeine (top left), poly-alginic acid (top right) and chitosan (bottom).
Although the most important caffeine source is from natural plants, it can also be artificially synthesized and added to certain foods. Some of the uses of caffeine range from improving mental alertness to being used as a cream for redness of skin [1]. Moreover, caffeine is usually formulated with pain killers to reduce pain.

As any drug, caffeine has to be consumed with moderation. One cup of brewed coffee contains between $75 \mathrm{mg}$ to $150 \mathrm{mg}$ of caffeine [1,4]. Previous studies [5] indicate that up to $400 \mathrm{mg}$ of caffeine is safe for healthy adult consumptions, which is about three or four cups of coffee. However, more than $500 \mathrm{mg}$ to $600 \mathrm{mg}$ of caffeine per day can cause side effects such as nervousness, insomnia, faster heart rate, addiction, amongst other side effects. Some people who are more sensitive to caffeine may feel side effects such as restlessness even from drinking one cup of tea. Children should limit intake of caffeine to under $100 \mathrm{mg}$ per day. How a person reacts to caffeine depends on regularity, age, mass, medication taking, and health conditions such as anxiety disorders. Although side effects start to emerge at around $600 \mathrm{mg}$ the toxicity level of caffeine has a Median Lethal Dose $\left(\mathrm{LD}_{50}\right)$ of 10 grams. From 2009 - 2011 the FDA reported only one death due to caffeine, although sixteen deaths have been reported from energy drinks from the FDA's center for food safety and applied nutrition. Reported cause of caffeine related deaths include dysrhythmias, seizures, and aspiration of emesis [6].

From a different perspective, caffeine effects have been studied on animals with remarkable discoveries. New Castle University has demonstrated that a low concentration of caffeine affects honeybee colonies. This report showed that the use of caffeine affected honeybees' long-term memory in a positive manner. Bees were able to remember the floral scent after up to $72 \mathrm{~h}$ [7]. Conversely, the same study showed

*Corresponding author: Abel E. Navarro, Science Department, Borough of Manhattan Community College, City University of New York, New York, USA, Tel: 001 212-2208000; Fax: 212-7488929; E-mail: anavarro@bmcc.cuny.edu

Received July 16, 2015; Accepted July 22, 2015; Published July 30, 2015

Citation: Zarzar A, Hong M, Llanos BP, Navarro AE (2015) Insights into the EcoFriendly Adsorption of Caffeine from Contaminated Solutions by Using Hydrogel Beads. J Environ Anal Chem 2: 150. doi:10.4172/2380-2391.1000150

Copyright: (C) 2015 Zarzar A, et al. This is an open-access article distributed under the terms of the Creative Commons Attribution License, which permits unrestricted use, distribution, and reproduction in any medium, provided the original author and source are credited. 
that when higher concentrations of caffeine were used, it acted as a repellent to pollinators [8]. This research concluded that caffeine could be used as a naturally-occurring pesticide.

One of the negative effects of caffeine in ecology was recently reported. This study showed that caffeine is present in rivers and oceans in concentrations ranging from 50 to $150 \mathrm{ng}$ per liter in the Pacific Northwest coast of the United States [9]. Studies showed that even $50 \mathrm{ng}$ per liter of caffeine are able to generate stress proteins in mussels. When an organism is stressed it generates stress protein to protect cells. Prolonged stress causes the shift of energy in a manner that organisms would continuously create stress proteins, rather than spend the energy on growth and reproduction [9]. This research also elucidated that higher concentrations of caffeine are found in less populated areas due to the lack of wastewater treatment plants $[9,10]$.

Based on the impact of caffeine on ecology, bioremediation has addressed proposes the use of biological materials (living and non-living) for the elimination of inorganic and organic pollutants. Previous work indicates that marine algae can be potentially used for the removal of copper (II) ions [11], sulfa drugs [12] and other heavy metals [13] from aqueous solutions. A more sustainable research has also demonstrated that spent chai tea leaves are good candidates for the removal of heavy metals from contaminates solutions [14] and artificial dyes [15-17]. More recent work has encapsulated spent tea leaves into chitosan hydrogel beads for the removal of cupric ions from solutions [18].

All these studies show that the use of bio-adsorbents is a "green", sustainable and inexpensive alternative to decontaminate organic and metal pollutants. The affinity of these biomaterials towards pollutants is associated with the presence of active organic functional groups such as carboxyl, amine, and hydroxyl groups, which have proven to be adsorption sites for inorganic and organic pollutants [11-18]. Other natural polymers are found in prevalent organisms in nature, such as chitosan and poly-alginic acid. Chitosan is a derivative of chitin which can be found in seashells. The abundance of seashells make chitosan a renewable adsorbent and a sustainable way to use waste seashells that line up shores and beaches [18].

Brown marine algae have demonstrated a high capacity to adsorb pollutants due to the functional groups with high electron densities [11-13]. These groups compose larger structures such as alginate and fucoidan [12,13]. Since the composition of marine algae has a high percentage of alginate, it was concluded that these groups are responsible for most of the adsorption [11]. Therefore, this study evaluates the role of experimental parameters such as $\mathrm{pH}$, hydrogel dose, salinity and time on the adsorption of caffeine from aqueous solutions by using chitosan $(\mathrm{CH})$ and alginate $(\mathrm{AB})$ hydrogel beads (structures shown in Figure 1). The experimental data not only proposes hydrogel beads as candidates for caffeine adsorption, but also create a new mindset in the risk of caffeine in the environment and alternative means to decrease its concentration in water bodies.

\section{Materials and Methods}

\section{Reagent and solutions}

Stock solution of $1000 \mathrm{ppm}(\mathrm{mg} / \mathrm{L})$ of caffeine was daily prepared by dissolving anhydrous caffeine (USP/FCC, Fisher Scientific) in deionized water. Solutions of different caffeine concentrations were made by dilution of this stock solution with deionized water. No buffer was used in the preparation of the solutions to minimize salt effect due to competition of active sites and solubility changes of caffeine in water. This procedure has been previously used with other inorganic and organic pollutants $[11,12]$. The initial solution $\mathrm{pH}$ of each caffeine solution was adjusted to the required values by adding small volumes of concentrated $\mathrm{HCl}$ and $\mathrm{NaOH}$ prior contact with the hydrogels beads. In the case of $\mathrm{CH}$, a $\mathrm{pH}$ value of 2 was not studied due to the solubility of chitosan in acidic media.

\section{Preparation of adsorbents}

Alginate hydrogel beads $(\mathrm{AB})$ were prepared according to a previously reported protocol [19]. In summary, a given mass of sodium alginate (Reagent grade, Spectrum Chemicals) was dissolved in deionized water, and left overnight under magnetic stirring. Upon dissolution, a $0.2 \mathrm{M}$ solution of calcium chloride was prepared with deionized water and also placed under magnetic stirring at room temperature. Then, by using a peristaltic pump, the alginate solution was pumped into the calcium solution drop by drop (Figure 2). Alginate beads $(\mathrm{AB})$ instantly formed and were kept under stirring for $12 \mathrm{~h}$ to allow the total cross-linking of calcium ions with alginate. Then, $A B$ were vigorously rinsed and suspended in deionized water; and stored at $4^{\circ} \mathrm{C}$ until its use in adsorption experiments. The average diameter of the produced $\mathrm{AB}$ adsorbents was $2 \mathrm{~mm}$.

Chitosan hydrogel beads $(\mathrm{CH})$ were prepared using the standard protocol [14]. Chitosan (Reagent grade, Spectrum Chemicals) was dissolved in a $4 \% \mathrm{v} / \mathrm{v}$ solution of glacial acetic acid in deionized water. Total dissolution was achieved after $24 \mathrm{~h}$ of magnetic stirring at room temperature. In a separate plastic container, a $2.5 \mathrm{M}$ solution of $\mathrm{NaOH}$ was prepared and kept under magnetic stirring. Similar to $\mathrm{AB}$ preparation, the acidic solution of chitosan was pumped into the $\mathrm{NaOH}$ solution by using a peristaltic pump, drop by drop. $\mathrm{CH}$ drops instantly formed due to neutralization of acetic acid with $\mathrm{NaOH}$. Then, $\mathrm{CH}$ were then rinsed and suspended in deionized water and stored in the refrigerator until its use.

\section{Determining the dry ratio of beads}

As any hydrogel, $\mathrm{AB}$ and $\mathrm{CH}$ have a high water to maintain their structure. However, adsorption properties are only attributed to the biopolymers that make both hydrogels and to the water. Prior studies with these $\mathrm{AB}$ and $\mathrm{CH}$ hydrogels determined the dry/wet mass ratio to report the actual biomass content per hydrogel bead [18]. In brief, different number of wet beads were weighed and placed in an oven at no more than $60^{\circ} \mathrm{C}$ during $12 \mathrm{~h}$. Then, the mass of the dried beads was recorded. A linear calibration curve was made to correlate the wet and dry mass and a conversion factor (f) was obtained as shown in Equation (1).

\section{Dry mass $x f=$ Wet mass $(1)$}

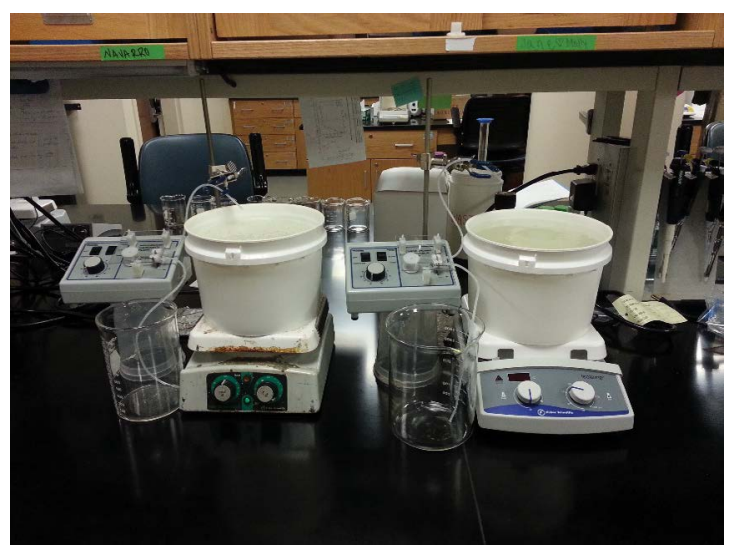

Figure 2: Preparation of alginate beads by cross-linking with $\mathrm{CaCl}_{2}$ solution. 


\section{Adsorption experiments}

Discontinuous experiments were carried out in duplicate at room temperature combining variable masses of $\mathrm{AB}$ and $\mathrm{CH}$ according to their dry/wet ratios. Adsorbents were mixed with $50 \mathrm{~mL}$ solution of caffeine of different concentrations (in $\mathrm{mg} / \mathrm{L}$ ) and incubated in an orbital shaker at $250 \mathrm{rpm}$ during $12 \mathrm{~h}$. This adsorption time was determined by preliminary experiments, which indicated that equilibrium is reached in less than $12 \mathrm{~h}$. Initial solution $\mathrm{pH}$ was adjusted depending on the type of effect to be studied. Thereafter, the mixtures were separated by gravity and the remaining caffeine concentration was determined through UV-vis spectrophotometry using a microplate reader (Synergy4, Biotek) at a wavelength of $284 \mathrm{~nm}$. This wavelength is the value at which caffeine reports is maximum ultraviolet radiation absorbance.

\section{Determination of the optimum $\mathrm{pH}$}

The role of the initial solution $\mathrm{pH}$ was studied between the values of two and eight to determine the highest adsorption for caffeine. The $\mathrm{pH}$ was adjusted by adding small volumes of $2 \mathrm{M}$ solutions of $\mathrm{HNO}_{3}$ and $\mathrm{NaOH}$, until reaching the $\mathrm{pH}$ desired. A control sample with no adsorbent was simultaneously prepared and shaken to compare the initial caffeine concentration. Solution $\mathrm{pH}$ of the samples were taken after adsorption, showing small variations. This indicates that uptake of caffeine and presence of adsorbents did not alter the solution $\mathrm{pH}$. The $\mathrm{pH}$ value, at which the highest adsorption was observed, was used in the following effects.

\section{Determination of the optimum hydrogel dose}

In order to determine the optimal mass which adsorbs caffeine at the highest rate, different hydrogel masses were tested at the optimum $\mathrm{pH}$. Hydrogel doses between 80 and $260 \mathrm{mg}$ were tested and combined with $50 \mathrm{~mL}$ of a $50 \mathrm{ppm}$ caffeine solution. The optimum hydrogel dose was determined and used in the salt effect.

\section{Effect of salts on the adsorption of caffeine}

A similar experiment was conducted but including three different salts: calcium nitrate $\left(\mathrm{Ca}\left(\mathrm{NO}_{3}\right)_{2}\right)$, sodium chloride $(\mathrm{NaCl})$ and sodium nitrate $\left(\mathrm{NaNO}_{3}\right)$ to determine the changes in the caffeine removal due to the presence of different salts.

\section{Determination of the minimum time to reach equilibrium}

A cost-effective technique should not only reduce materials expense, but also operational costs, which include energy and manpower use. For this reason, fast caffeine removal is desired to minimize extra expenses in the remediation of contaminated waters. For this experiments, a larger system was utilized, by preparing a 500 $\mathrm{mL}$ solution and titrating it to $\mathrm{pH} 7$ (neutral $\mathrm{pH}$ value). The solution was placed under magnetic stirring at $250 \mathrm{rpm}$. On the side, a given mass of $\mathrm{AB}$ and $\mathrm{CH}$ was weighed and added to the solution. A sample time 0 was taken prior addition of the adsorbents as a control sample. A timer was started as the adsorbents were added to the solutions. Smalls aliquots of the solutions were taken a different time intervals (according to a time table) and placed in smaller centrifuge tubes. Then, the residual caffeine concentration was determined by spectrophotometry.

\section{Data analysis}

Adsorption affinity of a given adsorbent can be quantified in different manners. One commonly used indicator is Removal Efficiency (\%RE) where initial and final caffeine concentrations are compared and expressed as percentage as shown in Equation (2).

$$
\% R E=\frac{\left(\mathrm{C}_{i}-\mathrm{C}_{f}\right)}{C_{i}} \times 100
$$

where $\mathrm{C}_{i}$ and $\mathrm{C}_{f}$ are the initial and residual caffeine concentrations. Data processing was conducted using Microsoft Excel and graphs were prepared using the statistical software, Origin V8.0. Error bars were added to the graphs to validate the statistical significance of the results.

\section{Results and Discussion}

\section{Role of $\mathrm{pH}$ on caffeine adsorption}

Experimental results are shown in Figure 3. In order to determine the optimal $\mathrm{pH}$ for the best caffeine adsorption rate, samples of different $\mathrm{pH}$ values ranging from two to eight were tested and analyzed. The results show us that $\mathrm{CH}$ beads have the best removal efficiency at a neutral $\mathrm{pH}$ of 7 . The graph also shows that acidic $\mathrm{pH}$ values do not promote the removal of caffeine for both hydrogel beads. This may be explained because at low $\mathrm{pH}$ values, caffeine (a Lewis base) gets protonated and does not interact with the electronically neutral $\mathrm{AB}$ biopolymer. An even more negative interaction would be observed with $\mathrm{CH}$ beads, which are expected to be positively charged, due to the protonation of amino groups at low $\mathrm{pH}$ values. It is important to highlight that $\mathrm{CH}$ was not studied at $\mathrm{pH} 2$, because of its high water solubility in acidic conditions. $\mathrm{AB}$ beads show a better removal efficiency at a $\mathrm{pH}$ of 6 , most likely due to the formation of hydrogen bonds between hydroxyl groups (from alginate) and basic nitrogen and oxygen atoms in caffeine. A similar hydrogen bond formation is expected to occur with $\mathrm{CH}$ at relatively higher $\mathrm{pH}$ values. However,

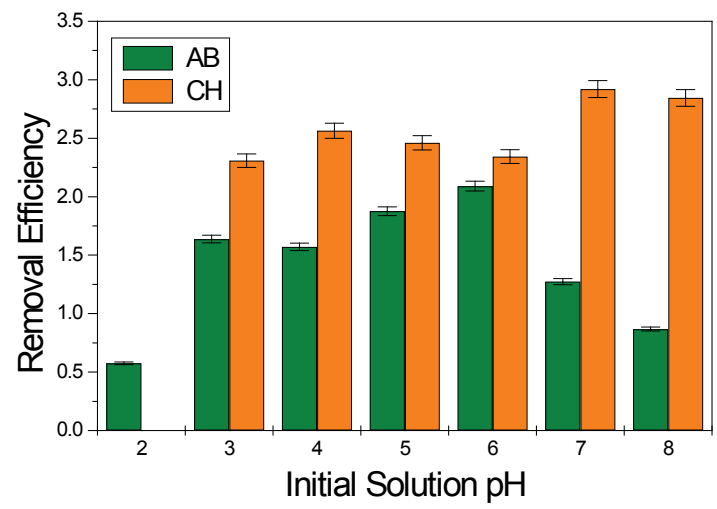

Figure 3: Effect of initial solution $\mathrm{pH}$ on the adsorption of caffeine.

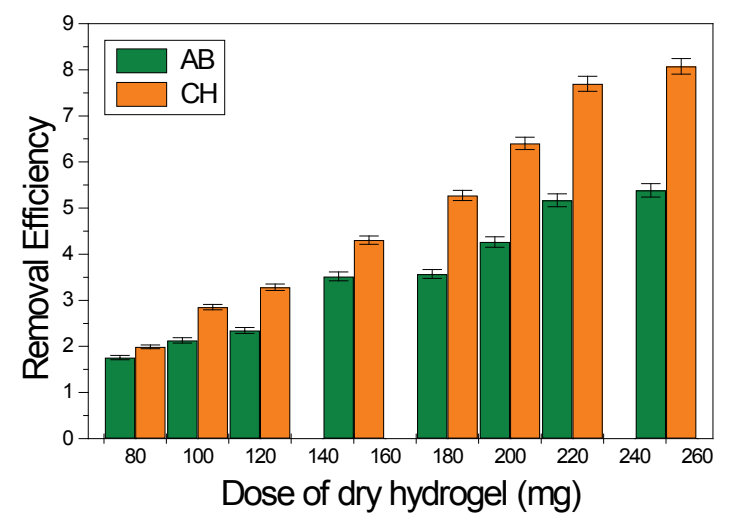

Figure 4: Effect of the hydrogel dose on the adsorption of caffeine. 
$\mathrm{CH}$ reports a higher adsorption, because chitosan, besides its hydroxyl groups, also has amino groups that can act as hydrogen bond donors and acceptors. These groups can enhance the adsorption capacity that is observed in $\mathrm{CH}$. From the results, it can be concluded that although $\mathrm{AB}$ and $\mathrm{CH}$ do not reach extremely high caffeine removal from solutions, but a strong acid-base effect is observed in solution. Future work includes the use of other adsorbents that are known to have chitosan-like functional groups.

\section{Minimum hydrogel dose for the removal of caffeine}

A cost-effect technique should minimize material consumption, therefore a maximum adsorption with the minimum mass of adsorbent is desired. As displayed in Figure 4, results show that as the dose of dry hydrogel increased, the more the caffeine was adsorbed from the solution. Further examining the removal efficiencies between $A B$ and $\mathrm{CH}$ beads, it can be seen that $\mathrm{CH}$ has better caffeine removal efficiency when compared to $A B$. Initially, with $80 \mathrm{mg}$ of $A B$ beads the adsorption rate is at about $1.7 \%$, while $\mathrm{CH}$ beads were at an even $2 \%$. At higher bead doses, close to $220 \mathrm{mg}$ of $\mathrm{AB}$, the adsorption rate reached about $5 \%$, whereas $\mathrm{CH}$ increased to $8 \%$. At even higher adsorbent masses $(260$ $\mathrm{mg}$ ) the removal efficiency kept increasing. However, the increase was not as substantial as the previous two masses. Therefore the small gain in caffeine removal is not justified by the addition of extra adsorbent. From these results, it was concluded that the optimum parameters for caffeine removal are at a $\mathrm{pH}$ of 7 using $\mathrm{CH}$ with the mass of 220 $\mathrm{mg}$, establishing the parameters for the next analyses. This increasing adsorption trends agrees with the increase in adsorption sites with higher hydrogel dose.

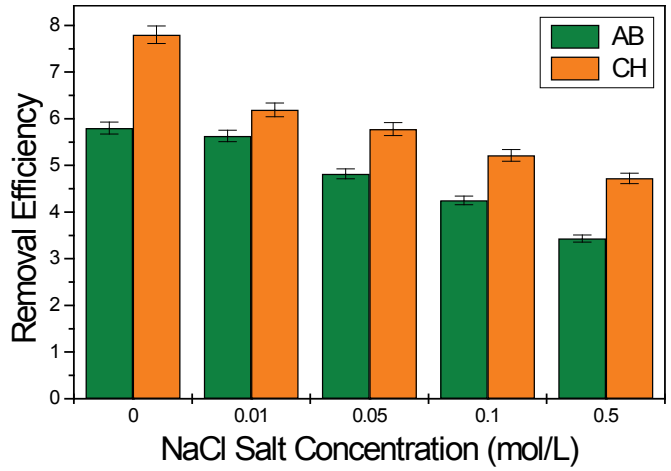

\section{Role of salts on the removal efficiency of caffeine}

Natural water resources such as oceans, lakes and rivers contain salts and other dissolved substances that might prevent or enhance the removal of caffeine. To address this task, three different salts were used to explore the role of salt in the adsorption. Experimental data is displayed in Figure 5. From the results, $\mathrm{CH}$ remained as the best adsorbent, but it was negatively affected by the presence of the three salts. Sodium chloride, sodium nitrate and calcium nitrate were chosen for this study due to their prevalence in seawater and surface waters in general $[11,12]$. Salt concentrations in the range of zero to $0.5 \mathrm{~mol} / \mathrm{L}$ were studied. As observed in Figure 5, the adsorption of caffeine decreases at increasing salt concentrations for both adsorbents. Apparently, AB hydrogels are able to resist the effect of salts better than $\mathrm{CH}$. This negative effect could be explained by the competition of active sites between caffeine and salt ions. This is confirmed by the fact that calcium nitrate shows a stronger effect with both hydrogels. At high calcium nitrate concentrations, the removal efficiency drops to values close to zero. This could be explained by the +2 charge of calcium ions, which may interrupt the caffeine/adsorbent interactions. This behavior has been also observed with other pollutants like drugs, dyes and heavy metals $[12,17,18]$.

\section{Minimum adsorption time to reach equilibrium}

Fast adsorption rates are not only practically attractive, but also reduce workforce and energy consumption. Therefore, determining the minimum adsorption time for the removal of caffeine is crucial. Experimental results are shown in Figure 6. The time-dependent experiment shows that after ten minutes, close to $85 \%$ of the adsorption is achieved and after twenty minutes the beads adsorb caffeine to their

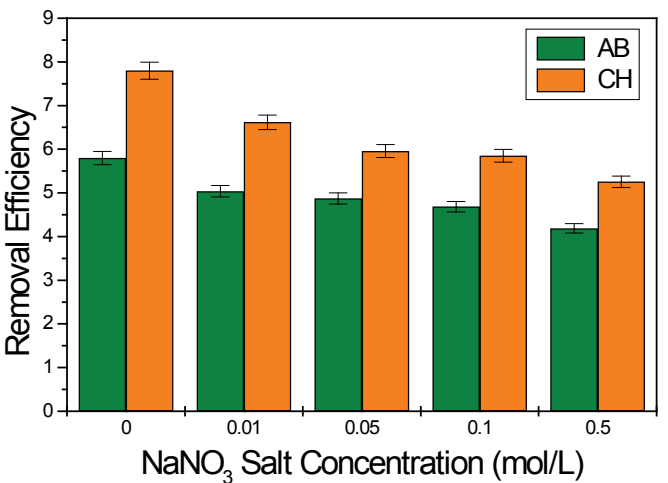

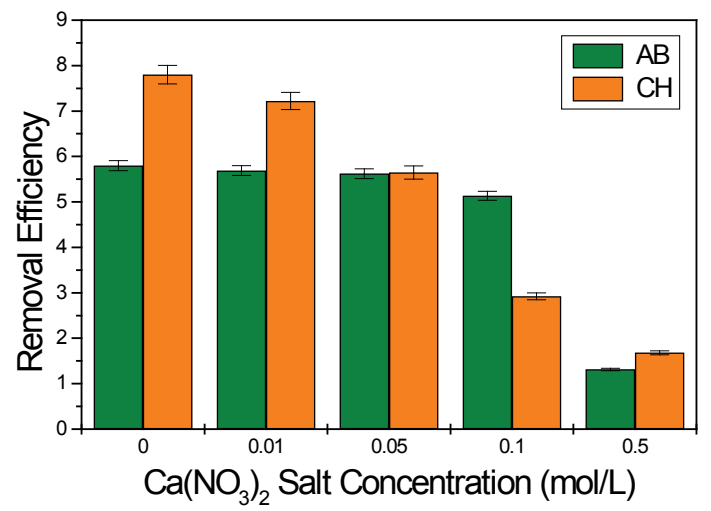

Figure 5: Effect of the salts on the adsorption of caffeine. Top left: $\mathrm{NaCl}$, Top right: $\mathrm{NaNO}_{3}$, and $\mathrm{Bottom} \mathrm{Ca}\left(\mathrm{NO}_{3}\right)_{2}$. 


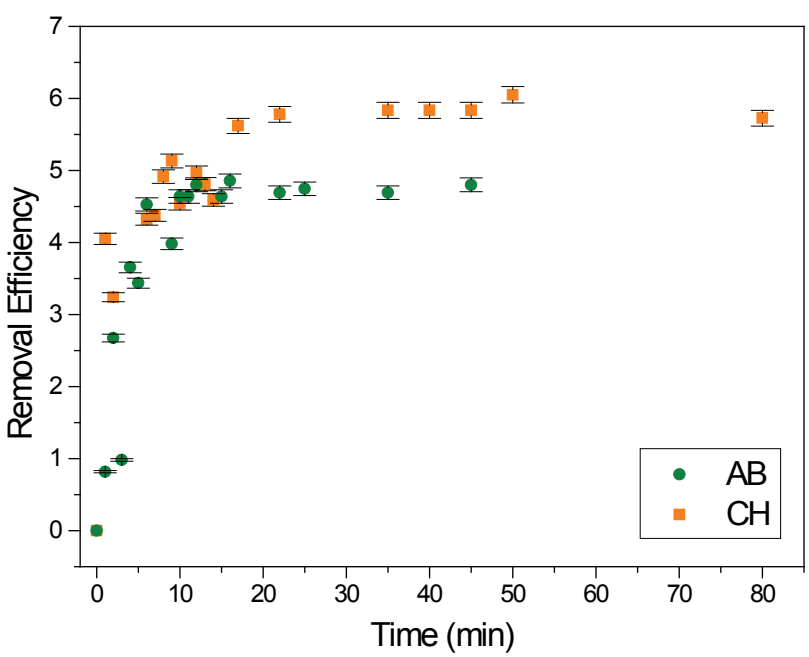

Figure 6: Time-dependence of the caffeine removal efficiency with hydrogel beads.

maximum capacity. The data corroborates that $\mathrm{CH}$ hydrogels have a better adsorption capacity when compared to $\mathrm{AB}$ hydrogels. However, a faster adsorption is observed for $\mathrm{AB}$ (less than 10 minutes). Perhaps this is the most valuable and important finding of the project, which although the adsorption is not stunningly high, but it reaches its highest value in a very short time (10 minutes).

\section{Conclusions}

The presence of drugstore products such as caffeine are a dormant problem in the environment. At this time, there are no specific regulations about the disposal of these products into the water bodies, but the ecological effects are more than evident. This research evaluates the use of hydrogel beads of alginate $(\mathrm{AB})$ and chitosan $(\mathrm{CH})$ as adsorbents of caffeine from aqueous solutions by studying experimental conditions that maximize its uptake. Experimental data shows us that $\mathrm{CH}$ is a better adsorbent than $\mathrm{AB}$, due to formation of hydrogen bonds with caffeine. Amongst the studied parameters, $\mathrm{pH}$ and salinity displayed the strongest effect on adsorption. A maximum removal efficiency of $8.4 \%$ was achieved with a mass of $220 \mathrm{mg}$ at a solution $\mathrm{pH}$ of 7 with $\mathrm{CH}$ hydrogel beads. On the other hand, salinity had a negative effect of the elimination of caffeine due to competition for active sites with small ions such as sodium and calcium ions. Although these materials are not able to totally remove caffeine from contaminated waters, nevertheless $\mathrm{CH}$ and $\mathrm{AB}$ reach adsorption equilibrium in less than 20 minutes. This study with hydrogel beads provides new alternatives for the elimination of pharmaceutical products and is expected to create a new eco-friendly mindset on caffeine disposal and regulation. Moreover, it can start new trends in materials science research for the development of more efficient, yet inexpensive and sustainable adsorbents.

\section{Acknowledgement}

This research was conducted under the financial support of the CSTEP and CUNY Research Scholars Program at BMCC. Authors would like to acknowledge Gabrielle Jonny for her technical expertise and participation in preliminary data and the Biotechnology Laboratory Facility at BMCC. A.N. would like to thank PNICP (project ECIP-1-P-042-14) for the financial sponsorship.

\section{References}

1. WebMD (2014) Caffeine: Uses, Side Effects, Interactions and Warnings.

2. Huff Post Green, Pacific Ocean Caffeine Pollution Analyzed In Recent Study. Posted on August 6th 2012. Last seen: July 15, 2015.

3. Trevor-Roper H (1982) Medicine in Politics. The American Scholar 51: 23-41.

4. US National Library of Medicine (2015) Caffeine in the diet: MedlinePlus Medical Encyclopedia.

5. Mayo Clinic (2015) Nutrition and Healthy Eating. Caffeine: How Much Is Too Much

6. Seifert SM, Schaechter JL, Hershorin ER, Lipshultz SE (2011) Health effects of energy drinks on children, adolescents, and young adults. Pediatrics 127 511-528.

7. Williamson SM, Wright GA (2013) Exposure to multiple cholinergic pesticides impairs olfactory learning and memory in honeybees. J Exp Biol 216: 1799-1807.

8. Wright GA, Baker DD, Palmer MJ, Stabler D, Mustard JA, et al. (2013) Caffeine in floral nectar enhances a pollinator's memory of reward. Science 339: 1202-1204.

9. Living on Earth (2012) Caffeine in Ocean Water.

10. Halden R (2010) Contaminants of emerging concern in the environment: Ecological and human health considerations. American Chemical Society, Washington DC, USA.

11. Reyes U, Navarro A, Lianos B (2009) Use of seaweed for biosorption of cupric ions from aqueous solution. Rev Soc Quim Peru 75 : 353-361.

12. Navarro A, Lim H, Chang E, Lee $Y$, Manrique A (2014) Uptake of Sulfa Drugs from Aqueous Solutions by Marine Algae. Sep Sci Technol 49: 2175-2181.

13. Fourest E, Volesky B (1996) Contribution of sulfonate groups and alginate to heavy metals biosorption by the dry biomass of Sargassum fluitans. Environ Sci Technol 30: 277-282.

14. Guibal E, Milot C, Tobin M (1998) Metal-anion sorption by chitosan beads: Equilibrium and kinetic studies. Ind Eng Chem Res 37: 1454-1849.

15. Park R, Kim G, Shen L, Hong M, Navarro A. (2014) Batch adsorption of heavy metals onto chai tea residues for the bioremediation of contaminated solutions. Current Topics in Biotechnol, 8: 51-62.

16. . Bellatin L, Herrera O, Navarro A, Sun R, Llanos B (2014) Study on the biosorption of basic yellow 57 dye, basic blue 99 and acid red 18 from hair dyes onto green tea leaves. Rev Soc Quim Peru, 80(1): 9-23.

17. Zahir H, Naidoo M, Kostadinova R, Ortiz K, Sun R, Navarro A (2014) Decolorization of hair dye by lignocellulosic waste materials from contaminated waters. Front Environ Toxicol 2: 28.

18. Choi Y, Isaac P, Irkakhujaev S, Masud M, Navarro A (2015) Use of Spent Tea Wastes-Chitosan Capsules for Removal of Divalent Copper lons. J J Environ Sci, 1: 003.

19. Kostadinova R, Sikorska G, Naidoo M, Navarro A (2014) Acid-base properties of the adsorption of synthetic dyes from solutions. J Environ Anal Toxicol 4: 240. 